\title{
www.lungenaerzte-im-netz.de Ein neuer Patienteninformationsdienst der Pneumologen geht im September online
}

\author{
New Patient Information Service will be Online this September
}

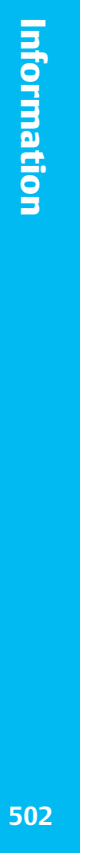

Anlässlich des diesjährigen, achten Deutschen Lungentags am 24.9.2005 startet im Internet ein neuer Patienteninformationsdienst der Lungenfachärzte, den die Deutsche Gesellschaft für Pneumologie (DGP) und der Bundesverband der Pneumologen (BdP) gemeinsam herausgeben. Ziel dieses Internetdienstes ist es, Patienten, Angehörige und medizinisch interessierte Laien umfassend über Lungen- und Atemwegserkrankungen zu informieren und pneumologische Themen einer breiteren Öffentlichkeit bekannt zu machen. Darüber hinaus bietet dieses Informationsportal ein deutschlandweites Fachärzteverzeichnis und damit den dort eingetragenen Lungenfachärzten und -Kliniken eine interessante Plattform für ihre Praxis- oder Klinik-Homepage. Durch die Einbettung in ein seriöses, fachlich fundiertes und tagesaktuelles, redaktionelles Umfeld eröffnet der Webauftritt den Pneumologen Möglichkeiten zur eigenen Öffentlichkeitsarbeit mit Hinweis auf fachärztliche Spezialisierungen, Praxisschwerpunkte oder spezielle Angebote.

\section{Die Inhalte: laienverständlich - anschaulich - multimedial}

Ein Kernstück des Internetangebots ist die Rubrik „Krankheiten A-Z“, in der die wichtigsten pneumologischen Erkrankungen umfassend und detailliert vorgestellt und hinsichtlich ihrer Ursachen, Diagnostik, Therapie- und Präventionsmöglichkeiten laiengerecht erklärt werden. Zu den Volkskrankheiten COPD, Asthma und Allergien gibt es zusätzliche Service-Teile, die Betroffene auf Patientenschulungsprogramme, Selbsthilfegruppen, Rehabilitations-, Sport- und Freizeitmöglichkeiten sowie auf praktische Tipps und nützliche Adressen für ihre persönliche Krankheitssituation hinweisen. Auch Themen wie Rauchen, Umweltschad- stoffe, Schlaf, Beatmung sowie die einzelnen pneumologischen Untersuchungsmethoden sind auf den Informationsbedarf betroffener Laien abgestimmt und werden allgemeinverständlich dargestellt. Dabei werden allerdings keine öden Textwüsten den PC-Schirm beherrschen, sondern die Beiträge sind übersichtlich und internetgerecht strukturiert. Inhalte werden multimedial durch Bilder, Grafiken, Computeranimationen, Video- oder Audiofiles illustriert. So sind zum Beispiel Aufbau und Funktion des Atemwegssystems in 3-D-Bildern, die schrittweise von den oberen Atemwegen bis zu den Alveolen führen, plastisch dargestellt. Auch Audiofiles, die typische Auskultationsgeräusche demonstrieren, und Videoclips, die dem Benutzer auf eine spannende und anschauliche Weise die Ursachen und Symptome einiger Krankheiten näher bringen, sind geplant.

Einen wichtigen Stellenwert auf der Website der Lungenärzteim-Netz hat die aktuelle Berichterstattung über Neuigkeiten aus der pneumologischen Forschung, Klinik und Praxis, Therapie und Rehabilitation sowie Empfehlungen der Fachgesellschaften und -verbände. Neben aktuellen Meldungen (5x wöchentlich) werden auf der Startseite auch fachlich relevante Fokusthemen über einen längeren Zeitraum präsentiert, die gerade im Brennpunkt der Diskussion stehen. Künftig soll es außerdem möglich sein, für jede Region in Deutschland die täglich aktualisierten Daten über Pollenflug, Ozonwerte und Feinstaubbelastung direkt auf der Website abzurufen. 


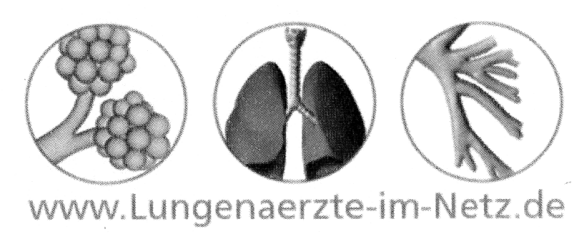

\author{
Ihre Ärzte/Klinik-Homepage \\ bei www.lungenaerzte-im-netz.de - \\ dem Informationsdienst für Ihre Patienten und Angehörige \\ herausgegeben von der Deutschen Gesellschaft für Pneumologie (DGP) und dem \\ Bundesverband der Pneumologen (BdP)
}

Vorteile für Ihre Homepage bei www.lungenaerzte-im-netz.de:

- Redaktionelles Umfeld mit mehr als 1.500 Seiten Informationen zu pneumologischen

Erkrankungen und vielen weiteren nützlichen Tipps

- Tägliche News zu aktuellen pneumologischen Themen

- Breite Bekanntmachung durch Fernsehen und Printmedien

\begin{abstract}
Kosten:
Einzelpraxis - ohne bestehende Homepage

Einzelpraxis - mit bestehender Homepage

$8 €$ / Monat

$4 €$ / Monat

Gemeinschaftspraxen - ohne bestehende Homepage

Gemeinschaftspraxen - mit bestehender Homepage

$10,00 € /$ Monat

$5,00 € /$ Monat

Kliniken - mit bestehender Homepage

$25,00 € /$ Monat

Hinzu kommt eine einmalige Erstellungsgebühr von $15 €$.

Alle Kosten verstehen sich zzgl. 16\% Mwst.
\end{abstract}

Kontakt und Information:

Herr Steven Monks

Tel: 089 / 64248212

Email: st.monks@monks.de

\section{Zentrale Informationsquelle für Patienten, Interessierte} und Presse

Für die Erstellung der Inhalte und technische Umsetzung ist ein Team aus Medizinern, Naturwissenschaftlern, Programmierern und Grafikern der Agentur „Sean Monks Ärzte-im-Netz“ zuständig. Ein Redaktionsgremium, das sich aus Vertretern der DGP und des BdP zusammensetzt, liest die erstellten Texte gegen, bevor sie online gestellt werden. Dies garantiert die hohe Qualität und fachliche Fundiertheit der Inhalte des Internetdiensts. Darüber hinaus bildet der Informationsdienst die Basis für eine erfolgreiche Öffentlichkeitsarbeit, um eine größere Aufmerksamkeit für pneumologische Themen zu erregen und diese verstärkt publik zu machen. „Sean Monks Ärzte-im-Netz“ verfügt neben einem eigenen medizinisches Bildarchiv über hervorragende Kontakte zu zahlreichen Fernsehredaktionen im Lande und ist langjähriger Partner von deutschen Presseagenturen. So können in enger Kooperation mit den Medien Fernsehbeiträge und Printartikel in Tagespresse, Wochenzeitungen oder Zeitschriften zum
Thema Lungen- und Atemwegserkrankungen produziert werden, die unter Nennung der Internetadresse www.Lungenaerzte-imNetz.de auf weiterführende Informationen für interessierte Laien hinweisen. Auf diese Weise wird eine breite Bekanntmachung und eine gesteigerte Nutzung des Internetdienstes erreicht.

\section{Medienpräsenz ist der Schluissel zum Erfolg}

Der gesamte Webauftritt ist frei von Werbung, da Neutralität auch eine entscheidende Voraussetzung für die enge Zusammenarbeit mit den Medien ist. Die Startfinanzierung wird vom Pharmaunternehmen GlaxoSmithKline geleistet, ansonsten finanziert sich der Informationsdienst über die Abonnementgebühren der Ärzte- und Klinik-Homepages.

Welchen Erfolg das Konzept der Agentur „Sean Monks Ärzte-imNetz“ beim Aufbau medizinischer Informationsportale für Laien hat, zeigt der Pressespiegel für den Internetdienst des Berufsver- 
bands der Kinder- und Jugendärzte (www.kinderaerzte-imnetz.de): Bisher sind in Printmedien mit einer Auflage von über 320 Millionen und in TV-Sendungen mit mehr als 80 Millionen Zuschauern Beiträge über pädiatrische Themen mit Hinweis auf die Kinderärzte-im-Netz erschienen. Mit dem gleichen Erfolgskonzept sind aufgrund des von der Agentur Monks aufgebauten Medien-Netzwerks vielfältige Möglichkeiten der Öffentlichkeitsarbeit realisierbar, um auch pneumologische Themen einer breiteren Öffentlichkeit bekannt zu machen - und das im Sinne der Berufsverbände und Fachgesellschaften.

Der Nutzen des Informationsportals für niedergelassene Pneumologen und Facharzt-Kliniken ist zweierlei: Zum einen dürften sie mit einer größeren Compliance ihrer Patienten rechnen können, da eine gründliche und fachlich korrekte Information die Mitwirkung und Zuverlässigkeit von Betroffenen und Angehörigen während diagnostischer, therapeutischer und präventiver Maßnahmen merklich verbessern kann. Zum anderen bietet der Internetdienst Lungenärzte-im-Netz Pneumologen eine kostengünstige, seriöse und öffentlichkeitswirksame Plattform für ihre persönliche Homepage. Ein deutschlandweites Fachärzte-Verzeichnis auf der Startseite des Patienteninformationsportals bietet verschiedene Suchmöglichkeiten, um Fachkliniken und Ärzte (pro Fachbereich oder Fachgebietsschwerpunkt) anhand ihres Namens, des Ortes ihrer Praxis/Klinik oder per Stichworteingabe zu finden.

Die monatlichen Abonnementkosten für das Einstellen einer Homepage betragen $8,00 €$ für Einzelpraxen und $10,00 €$; für Gemeinschaftspraxen. Die Anbindung einer bereits bestehenden Homepage ist noch günstiger und beläuft sich auf $4,00 €$ für Einzelpraxen, 5,00 für Gemeinschaftspraxen sowie 15,00€; für Kliniken. Im Preis inbegriffen sind dabei die folgenden Leistungen: Individuelle E-mail-Adressen und Unterverzeichnisse machen einzelne Ärzte auch in Gemeinschaftspraxen oder Kliniken adressierbar. Kleinere Änderungen bzw. Aktualisierungen (zum Beispiel der Praxis-Öffnungszeiten) lassen sich über einen passwortgeschützten Online-Zugang kinderleicht und selbständig vornehmen. Die Homepages werden bei externen Suchmaschinen angemeldet und sind damit gut auffindbar, auch eine Besucherstatistik ist abfragbar. Jede Homepage kann bis zu 40 Seiten umfassen. Kontaktformulare für Patientenanfragen per E-mail und Gästebücher können auf Wunsch (und ohne Mehrkosten) eingerichtet werden. Zur Bebilderung steht ein umfangreiches Fotoarchiv zur Verfügung. Die Eintragung einer Praxis in mehrere Fachbereich-Kategorien zugleich ist grundsätzlich möglich. Auch eine Anfahrtsskizze wird (in Kooperation mit dem Routenplaner-Anbieter Map24) für jede Homepage erstellt. Für Fragen und weitere Informationen (zum Beispiel zum Layout) steht wochentags ein Servicemitarbeiter per Telefon (089 / 642482 12) und E-mail (st.monks@monks.de) bereit.

\author{
Seltene Lungenkrankheiten, Teil 3 \\ Kirsten D, Costabel U (Hrsg.) \\ Großhansdorf: Inter-Pneu-Verlag, 2005; 183 S., 48 Abb., 30 Tab. \\ $€ 32$,-. ISBN 3-9 810132-0-4
}

Das vorliegende Buch ist der 3. Band der Reihe: „Seltene Lungenkrankheiten“. Acht Krankheitsbilder werden dargestellt, die auf Vorträge anlässlich der DGP-Kongresse in München und Frankfurt zurückgehen.

Der Bogen spannt sich von interstitiellen Lungenkrankheiten (Akute interstitielle Pneumonie, „non-specific interstitiell pneumonia“ NSIP) und der verwandten Vaskulitis (mikroskopische Polyangiitis) über eine seltene Differenzialdiagnose der pulmonalen Hypertonie, die pulmonal veno-occlusive Erkrankungen hin zu Raritäten wie der Bronchitis plastica.

Klinisch wichtig und gut dargestellt ist bei Kindern und jungen Erwachsenen der Formenkreis der primär zilären Dyskinesie. Der Autor konnte sich allerdings vermutlich mangels Verfügbarkeit und eigener Erfahrung nicht mit nuklearmedizinischen Methoden zur Messung der mukoziliären Clearance anfreunden. Weiterhin ist die Darstellung der „Vocal cord dysfunction“ bedeutsam, da dieses Krankheitsbild zunehmend häufiger in differenzialdiagnostische Überlegungen eines schwer zu therapierenden Asthma's einbezogen wird.

Betont werden soll ein sehr interessanter Beitrag zur BCG-itis. Die Aktualität dieses Krankheitsbildes entsteht durch die zunehmende Anwendung von BCG-Instillationen zur Therapie von Blasenkarzinomen.

Die einzelnen Kapitel sind sehr übersichtlich. Fallbeispiele, zahlreiche Abbildungen und Tabellen tragen dazu bei, dass das Buch leicht zu lesen ist. In Verbindung mit ausführlichen Literaturangaben stellt das Buch ein wertvolles Nachschlagewerk dar, was bei der Erfahrung und Reputation der Autoren nicht anders zu erwarten war, und unterstützt insbesondere das „daran denken“.

P. Haidl, Schmallenberg 